\title{
THERMOTROPIC, REFRACTING AND THERMO-OPTICAL PROPERTIES IN THREE HOMOLOGS OF 4-N-ALKYL-4'- CYANOBIPHENYLS
}

\author{
A. Nesrullajev \\ Department of Physics, Faculty of Science, Mugla Sitki Koçman University, 48000 Kötekli Muğla, Turkey \\ E-mail: arifnesr@mu.edu.tr
}

Received 1 October 2014; revised 25 November 2014; accepted 10 December 2014

\begin{abstract}
Investigations of temperature behaviour of the mean refractive index $n$, ordinary $n_{\mathrm{o}}$ and extraordinary $n_{\mathrm{e}}$ refractive indices, and birefringence $\Delta n$ have been carried out for three homologues of 4-n-alkyl-4'-cyanobiphenyls $(\mathrm{n}=8,10,12)$. The principal polarizabilities $\alpha_{0}$ and $\alpha_{\mathrm{e}}$, effective geometry parameter $\alpha_{\mathrm{eg}}$ and average polarizability $\alpha_{\text {ave }}$ have been calculated using the isotropic internal field model (Vuks approach). Temperature behaviour of the order parameter in regions of the smectic A-nematic, nematic-isotropic liquid and smectic A-isotropic liquid is discussed. All of the optical and orientational parameters, which have been obtained in this work, are in good agreement with the theoretical approach.
\end{abstract}

Keywords: liquid crystals, refractive properties, optical birefringence, phase transitions

PACS: 42.25.Lc; 42.70.Df; 64.70.M

\section{Introduction}

Liquid crystals (LCs) are partially ordered and physical anisotropic materials. These materials are very sensitive to different external effects, e. g. electric, magnetic and thermic fields, surfaces, boundary conditions, flows etc. Most applications of these materials in LCs technique and LCs technology depend upon their thermo-optical, electro-optical and magnetooptical properties [1-4]. These properties exhibit a very interesting behaviour in both regions of liquid crystalline mesophases and the regions of phase transitions. For the technical and technological applications of LCs, information about the optical anisotropy, refractive and polarization properties, and also about their temperature behaviour is important.

The knowledge of the optical anisotropy and the refractive indices $n, n_{\mathrm{o}}$, and $n_{\mathrm{e}}$ of liquid crystalline mesophases is essential for calculating of the order parameter of mesophases and polarizabilities of molecules [5-9]. Such calculations using the Vuks model (Vuks approach) [10, 11] or using the Neugebauer model (Neugebauer approach) have been made [12]. In the Vuks approach the isotropic internal field model and in the Neugebauer approach the anisotropic internal field models are taken into consideration. These two approaches lead to values of the refractive properties and order parameter of liquid crystalline mesophases, which are in sufficiently good agreement [5, $4,13-15$. Besides, one of the most known methods for calculating the order parameter without considering of the internal field is the Haller approximation method [16-18]. In this method the orientational order parameter can be determined only from the refractometric dates of liquid crystalline mesophase.

In this work the objects of investigations were three homologues of 4-n-alkyl-4'-cyanobiphenyls $(\mathrm{n}=8,10,12) .4$-n-alkyl-4'-cyanobiphenyls are stable mesogenic compounds, exhibit smectic A and nematic mesophases and have good (photo)chemical stability. Therefore they are sufficiently interesting objects for scientific investigations and very perspective materials for technical and technological applications. Numerous reports on the optical properties of the above-mentioned mesogenic compounds are known. Namely, phase transformations for 6CB, 7CB, $8 \mathrm{CB}$ and $10 \mathrm{CB}$ by positron annihilation spectroscopy were studied in [19]; temperature dependences of the optical birefringence and refractive indexes for $5 \mathrm{CB}$, $6 \mathrm{CB}, 7 \mathrm{CB}$ and $8 \mathrm{CB}$ were investigated in [20]; the frequency dependences of the refractive indexes for $5 \mathrm{CB}$ in the $\mathrm{THz}$ frequency range were investigated in [21]; 
the optical birefringence of $12 \mathrm{CB}$ at the $\mathrm{p}-\mathrm{Al}_{2} \mathrm{O}_{3}$ interface was investigated in [22]; the temperature dependences of the refractive indices and transmission losses for $5 \mathrm{CB}$ were studied in [23]; the optical birefringence for $5 \mathrm{CB}, 6 \mathrm{CB}, 7 \mathrm{CB}$ and $8 \mathrm{CB}$ were studied in [24]. But comparative investigations of the refracting and birefringent properties for high homologues of 4 -n-alkyl-4'-cyanobiphenyls $(\mathrm{n}=8,10,12)$ have not been carried out. Besides, comparative investigations of the connection between the refractive and birefringent properties with the polarizabilities (principal polarizabilities, average polarizability and effective geometry parameter) in 4-n-alkyl-4'-cyanobiphenyls have not been studied.

In this work, behaviour of the mean refractive index $n$, ordinary $n_{\mathrm{o}}$ and extraordinary $n_{\mathrm{e}}$ refractive indices, and birefringence $\Delta n$ have been studied in a large temperature region, especially in the regions of the smectic A-nematic, nematic-isotropic liquid and smectic A-isotropic liquid, for three homologues of 4 -n-alkyl-4'-cyanobiphenyls $(\mathrm{n}=8,10,12)$. Based on these dates, the order parameter $Q$, principal polarizabilities $\alpha_{0}$ and $\alpha_{\mathrm{e}}$, average polarizability $\alpha_{\text {ave }}$ and effective geometry parameter $\alpha_{\text {eg }}$ have been determined, using the isotropic internal field model.

\section{Theoretical background}

LCs are physically anisotropic materials and are characterized by various optical, diamagnetic, dielectric, viscous-elastic etc. parameters. Optical parameters of liquid crystalline materials include the refractive index $n$, ordinary refractive index $n_{\mathrm{o}}$, extraordinary refractive index $n_{\mathrm{e}}$ and the birefringence $\Delta n$.

The refractive index $n$ is related with the ordinary and extraordinary refractive indices as $[10,11,25,26$

$$
n^{2}=\frac{n_{\mathrm{e}}^{2}+2 n_{\mathrm{o}}^{2}}{3} .
$$

On the other hand, the ordinary and extraordinary refractive indices can be obtained as a function of the refractive index and birefringence as [8, 27, 28]

$$
\begin{aligned}
& n_{\mathrm{e}}=n-\frac{1}{3} \Delta n, \\
& n_{\mathrm{o}}=n+\frac{2}{3} \Delta n .
\end{aligned}
$$

The optical birefringence is an important parameter for determination of the orientational order parameter of LCs. In [2, 28-34] it is shown that the character of temperature dependences of the $\Delta n$ corresponds to the character of temperature transformation of the macro- scopic order parameter $Q$ of LCs. Besides, the character of the $\Delta n$ in the oriented smectic A and nematic mesophases is in good conformity with the theoretically predicted character of the temperature dependences of this order parameter [20, 24, 29, 35. 36-39

The parameter $Q$ is related with $\Delta n$ as $[24,34,35$.

$$
Q=\frac{\Delta n}{\Delta n_{0}} \text {. }
$$

Here $\Delta n_{0}$ is the birefringence of liquid crystalline material in the crystalline state (at $T=0$ ). At this temperature $Q=0$ takes place. Because the $\Delta n_{0}$ is a constant value for each liquid crystalline material, $Q \sim \Delta n$ takes place. Thus, the character of temperature dependences of the $\Delta n$ corresponds to the temperature dependences of the $Q$ parameter. We would like to note that several tensorial properties such as the anisotropy of dielectrical, magnetical, optical and elastical properties can be used for the determination of the macroscopic order parameter in LCs. Besides, as it is noted in [7, 21, 28, study of the refractive indexes as well as the birefringence is key for fundamental studies and practical applications of LCs.

In the model, presented in [10, 11], it is accepted that the local field in crystals is the same in all directions, i. e. that this field is isotropic. In this case (socalled Vuks relations) [10, 11, 33.

$$
\begin{aligned}
& \alpha_{\mathrm{e}}=\frac{3}{4 \pi N} \frac{n_{\mathrm{e}}^{2}-1}{n^{2}+2}, \\
& \alpha_{\mathrm{o}}=\frac{3}{4 \pi N} \frac{n_{\mathrm{o}}^{2}-1}{n^{2}+2}
\end{aligned}
$$

take place. Here $N$ is the number of liquid crystalline molecules per $\mathrm{cm}^{3}$. Taking into consideration that $\frac{3}{4 \pi N}$ is a constant value, Eqs. $6 \mathrm{a}$ and $6 \mathrm{~b}$ can be expressed as

$$
\begin{aligned}
& k \cdot \alpha_{\mathrm{e}}=\frac{n_{\mathrm{e}}^{2}-1}{n^{2}+2}, \\
& k \cdot \alpha_{\mathrm{o}}=\frac{n_{\mathrm{o}}^{2}-1}{n^{2}+2} .
\end{aligned}
$$

Thus, using temperature dependences of $n_{\mathrm{e}}, n_{0}$ and $n$, the $k \cdot \alpha_{\mathrm{e}}$ and $k \cdot \alpha_{0}$ values can be determined. If, however, the local field is anisotropic, Neugebauer's relations

$$
\begin{aligned}
& n_{\mathrm{e}}^{2}-1=\frac{4 \pi N \alpha_{\mathrm{e}}}{1-N \alpha_{\mathrm{e}} \gamma_{\mathrm{e}}}, \\
& n_{\mathrm{o}}^{2}-1=\frac{4 \pi N \alpha_{\mathrm{o}}}{1-N \alpha_{\mathrm{o}} \gamma_{\mathrm{o}}}
\end{aligned}
$$


can be used [12]. Here $\gamma_{\mathrm{e}}$ and $\gamma_{\mathrm{o}}$ are the internal field factors. Between the $\gamma_{\mathrm{e}}$ and $\gamma_{\mathrm{o}}$ factors the $\gamma_{\mathrm{e}}+2 \gamma_{0}=4 \pi$ connection takes place. Because the order parameter in LCs is temperature dependent, the $\gamma_{\mathrm{e}}$ and $\gamma_{\mathrm{o}}$ factors must also be temperature dependent. Therefore, Eqs. (8a) and (8b) may be written as [33, 40]

$$
\frac{1}{\alpha_{\mathrm{e}}}+\frac{2}{\alpha_{\mathrm{o}}}=\frac{4 \pi N}{3}\left(\frac{n_{\mathrm{e}}^{2}+2}{n_{\mathrm{e}}^{2}-1}+2 \frac{n_{\mathrm{o}}^{2}+2}{n_{\mathrm{o}}^{2}-1}\right) .
$$

Additionally, between $n, \alpha_{e}$ and $\alpha_{\mathrm{o}}$ the following connection takes place $[16,33,40]$ :

$$
\alpha_{\mathrm{e}}+2 \alpha_{\mathrm{o}}=\frac{9}{4 \pi N}\left(\frac{n^{2}-1}{n^{2}+2}\right) .
$$

Thus, knowing the $n_{\mathrm{e}}, n_{\mathrm{o}}$ and $n$ values, the $\alpha_{\mathrm{e}}$ and $\alpha_{\mathrm{o}}$ values, and also the polarizability anisotropy $\left(\alpha_{\mathrm{e}}-\alpha_{\mathrm{o}}\right)$ can be determined.

Ratio between $n_{\mathrm{o}}$ and $n_{\mathrm{e}}$ determines the value of the effective geometry parameter $\alpha_{\text {eg }}$ for liquid crystals with the positive optical anisotropy as [28, 41, 42]

$$
\alpha_{\mathrm{eg}}=\frac{n_{0}}{n_{\mathrm{e}}} .
$$

Because $n_{\mathrm{o}}$ and $n_{\mathrm{e}}$ are dependent on temperature, $\alpha_{\mathrm{eg}}$ are also dependent on temperature. $\alpha_{\mathrm{eg}}$ is an important parameter to understand the light deflection in liquid crystals. In [43, 44] it is shown that $\alpha_{\mathrm{eg}}$ is connected with light travelling near disclinations and other topological defects in liquid crystals, and with the orientation of the director field.

In $[5,6,28,33,36,37,40]$ temperature dependences of $n_{\mathrm{e}}, n_{0}, Q, \alpha_{\mathrm{e}}, \alpha_{\mathrm{o}}, \alpha_{\mathrm{eg}},\left(\alpha_{\mathrm{e}}-\alpha_{\mathrm{o}}\right), \gamma_{\mathrm{II}}, \gamma_{\perp}$ and $\left(\gamma_{\mathrm{II}}-\gamma_{\perp}\right)$ for various LCs have been determined and the connection between these parameters has been studied, using both the isotropic internal field model (Vuks approach) and the anisotropic internal field model (Neugebauer's approach). Liquid crystalline materials, which display smectic $\mathrm{C}$, smectic $\mathrm{A}$, nematic and cholesteric mesophases, have been used. In these works sufficiently good agreement between these values, estimated from these two independent methods, has been found.

\section{Experimental}

\subsection{Materials}

In this work, three even homologues of the 4-nalkyl-4'-cyanobiphenyls series were objects of our investigations. The homologues investigated are 4-noctyl-4'-cyanobiphenyls (8CB), 4-n-decyl-4'-cyanobiphenyls (10CB), and 4-n-dodecyl-4'-cyanobiphenyls
(12CB). 4-n-alkyl-4'-cyanobiphenyls were purchased from Merck and used without further purification. The structural formulae of 4-n-alkyl-4'-cyanobiphenyls are given in the Diagram. These liquid crystalline materials have uniaxial molecular symmetry, are colorless, thermally stable and stable to moisture. Besides, these materials have low-temperature liquid crystalline states, display enantiotropic mesophases and thermotropic phase transitions, and also have high positive optical anisotropy.

Diagram. The structural formulae of 4-n-alkyl-4'-cyanobiphenyls. 8CB: $\mathrm{n}=8 ; 10 \mathrm{CB}: \mathrm{n}=10 ; 12 \mathrm{CB}: \mathrm{n}=12$.

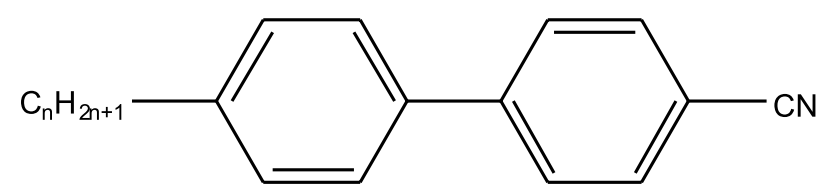

\subsection{Methods}

In this work, the temperature dependences of the refractive indexes $n, n_{\mathrm{e}}$ and $n_{\mathrm{o}}$ for $8 \mathrm{CB}, 10 \mathrm{CB}$ and $12 \mathrm{CB}$ have been measured. For these measurements the polythermic refractometry setup, based on an Abbe's refractometer, has been used. Accuracy for the refractive indexes measurements was as $0.1 \%$. The temperature changes of an Abbe's refractometer have been carried out using a recirculation immersion thermostat Ultraterm 200. Temperature of LCs under investigation was controlled by a digital temperature controller with an accuracy of $\pm 0.1 \mathrm{~K}$. A sketch of the experimental setup is presented in Fig. 1 .

The thermotropic and thermo-morphologic properties, and temperatures of phase transitions in $8 \mathrm{CB}, 10 \mathrm{CB}$ and $12 \mathrm{CB}$ were studied using the polarizing optical microscopy technique (POM). As it is well known, the POM is a sufficiently convenient and informative method for investigation of the



Fig. 1. Sketch of the experimental setup: (1) light source; (2) Abbe's refractometer; (3) digital temperature controller; (4) recirculation immersion thermostat; (5) polarizer. 
mesomorphic and morphologic properties of liquid crystals and also for identification of liquid crystalline mesophases.

For determination of the refractive indexes $n_{\mathrm{e}}$ and $n_{\mathrm{o}}$, peculiarities of polarizers, and homeotropic and planar alignment of liquid crystalline materials have been used. To obtain information on the alignment in a liquid crystalline state, the prisms of the refractometer were treated. The deposition of the mixture of $0.1 \%$ cetyl-trimethylammonium bromide in deionized and bidistilled water or the mixture of $1 \%$ lecithin in ethyl alcohol on the prisms provided the homeotropic orientation (yielding $n_{\mathrm{e}}$ ) of $8 \mathrm{CB}, 10 \mathrm{CB}$ and 12CB. The deposition of a film of polyvinylalcohol, that was subsequently rubbed with velvet tissue, on the prisms induced the planar alignment (yielding $n_{\mathrm{o}}$ ) of LCs under investigations. Degree of the homeotropic alignment was checked on control samples by the POM and estimations of the conoscopic pictures. The estimations showed that the mixture of $1 \%$ lecithin in ethyl alcohol provided better alignment than the mixture of $0.1 \%$ cetyl-trimethylammonium bromide in deionized and bidistilled water. In Fig. 2, the conoscopic pictures for these two cases are presented. As seen in this figure, the mixture of lecithin provides better homogeneity of the homeotropic alignment. Homogeneity of the planar alignment has been examined by the POM and estimated by the optical polarization (OP) degree. The value of the OP degree has been determined as

$$
P=\frac{I_{\max }-I_{\min }}{I_{\max }+I_{\min }} .
$$

Here the $I_{\min }$ is the intensity of light, transmitted from the sample, which was placed parallel to the polarizer (or analyzer); the $I_{\max }$ is the intensity of light, which was transmitted from the sandwich-cell, placed under $45^{\circ}$ to the polarizer (or analyzer). The degree of the planar orientation for $8 \mathrm{CB}, 10 \mathrm{CB}$ and $12 \mathrm{CB}$ was estimated as $P \approx 0.90-0.92$.

\section{Results and discussion}

Temperatures of phase transitions in $8 \mathrm{CB}, 10 \mathrm{CB}$ and $12 \mathrm{CB}$ were examined by observing of texture transformation, using the POM method. Heating was done at the rate of $1.0 \mathrm{~K} / \mathrm{min}$. Results of the examination are presented in the Table.

Table. Temperatures of phase transitions in $8 \mathrm{CB}, 10 \mathrm{CB}$ and $12 \mathrm{CB}$.

\begin{tabular}{c|cccc}
\hline \multirow{2}{*}{ Sample } & \multicolumn{4}{|c}{ Phase transition temperatures, K } \\
\cline { 2 - 5 } & Cr-SmA & SmA-N & N-I & SmA-I \\
\hline $8 \mathrm{CB}$ & 294.3 & 306.8 & 313.8 & - \\
\hline $10 \mathrm{CB}$ & 317.1 & - & - & 323.5 \\
\hline $12 \mathrm{CB}$ & 321.3 & - & - & 331.7 \\
\hline
\end{tabular}

The temperature dependences of the refractive indices $n, n$ and $n_{0}$ for $8 \mathrm{CB}, 10 \mathrm{CB}$ and 12CB are presented in Figs. $3-5$. As seen in these figures, temperature dependences of $n$ for liquid crystals under investigation exhibit practically linear behaviour with slight fluctuations in the region of the phase transitions. However, as the temperature increases, the refractive indices $n_{\mathrm{e}}$ and $n_{\mathrm{o}}$ show different behaviour. The refractive index $n_{\mathrm{e}}$ strongly depends on the temperature and decreases in the smectic A mesophase range with an increase in temperature for $10 \mathrm{CB}$ and $12 \mathrm{CB}$. The refractive index $n_{\mathrm{o}}$ shows weak temperature dependence in the mesophase region but some increase of this index near the clearing temperature for these liquid crystals. In the smectic A-isotropic liquid (SmA-I) phase transition region, a disappearance of the refractive indices $n_{\mathrm{e}}$ and $n_{\mathrm{o}}$ takes place (Figs. 4, 5). This effect is connected with disappearance of the optical anisotropic properties and appearance of the optical isotropic properties in liquid crystalline materials at the clearing temperature. Then, in the isotropic liquid state the refractive index $n$ decreases slightly with an increase in temperature, like other liquid crystalline materials for the nematicisotropic liquid (N-I) and cholesteric-isotropic liquid (Ch-I) phase transitions [33, 37, 40, 45]. (a)

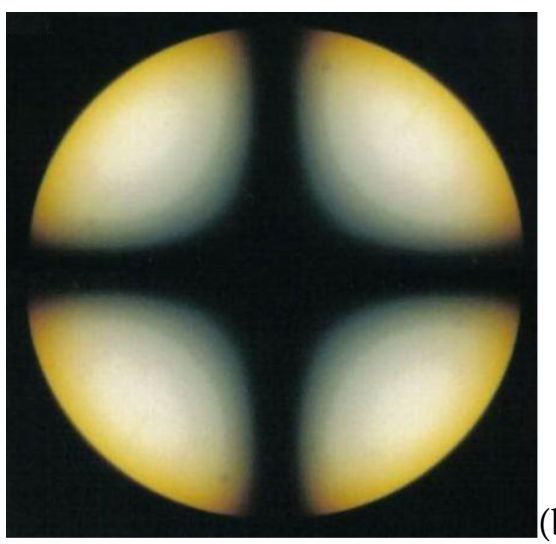

(b)

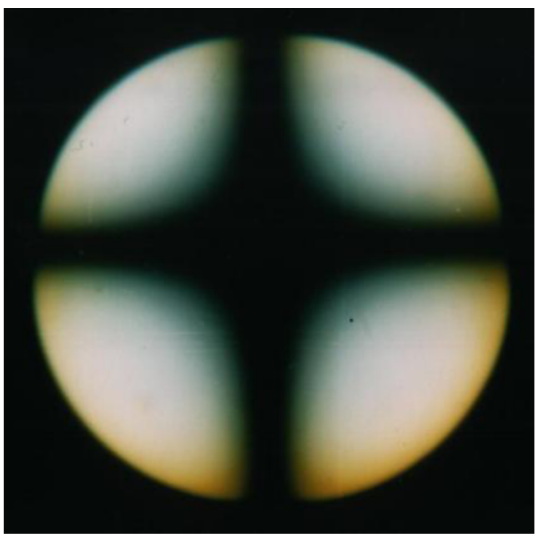

Fig. 2. Conoscopic pictures of homeotropic oriented samples: (a) orientation by lecithin; (b) orientation by cetyl-trimethylammonium bromide. 


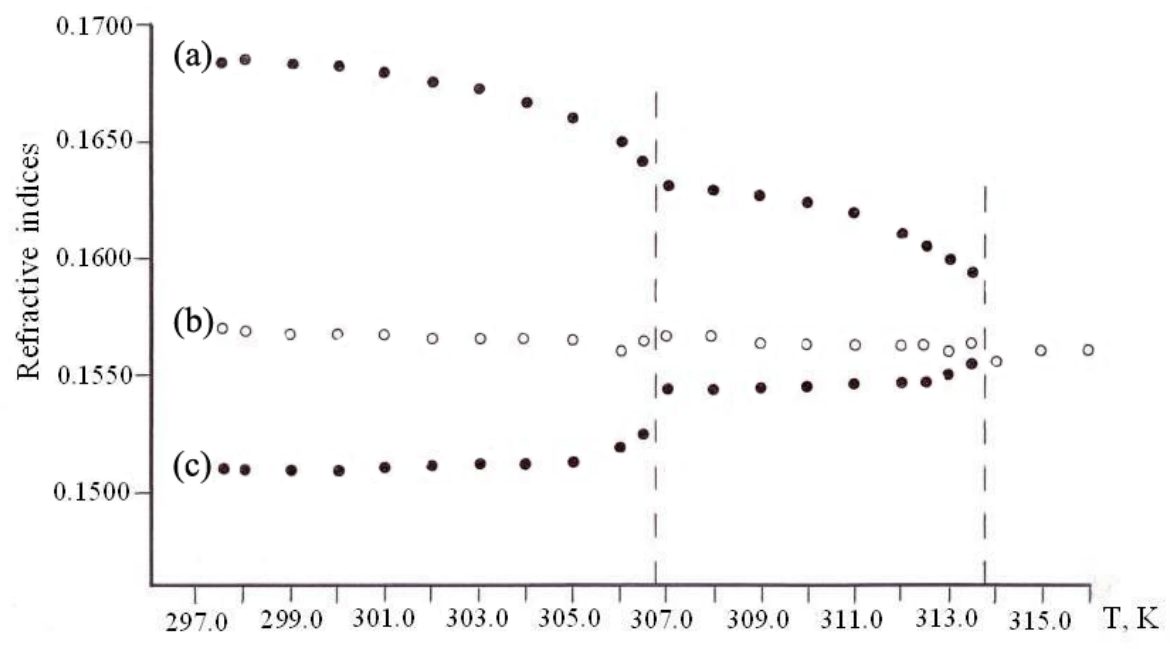

Fig. 3. The temperature dependences of the $n_{\mathrm{e}}(\mathrm{a})$, $n$ (b) and $n_{\mathrm{o}}$ (c) refractive indexes for $8 \mathrm{CB}$.

As seen in Fig. 3, the refractive indices $n_{\mathrm{o}}$ and $n_{\mathrm{e}}$ undergo abrupt changes twice: in the smectic A-nematic $(\mathrm{SmA}-\mathrm{N})$ and $\mathrm{N}-\mathrm{I}$ phase transition regions. A similar interesting behaviour of the refractive indexes for liquid crystals in the smectic A-cholesteric (SmA-Ch) and cholesteric-isotropic liquid (Ch-I) phase transitions was also observed in [33, 40]. Such character of the temperature dependences of the above-mentioned
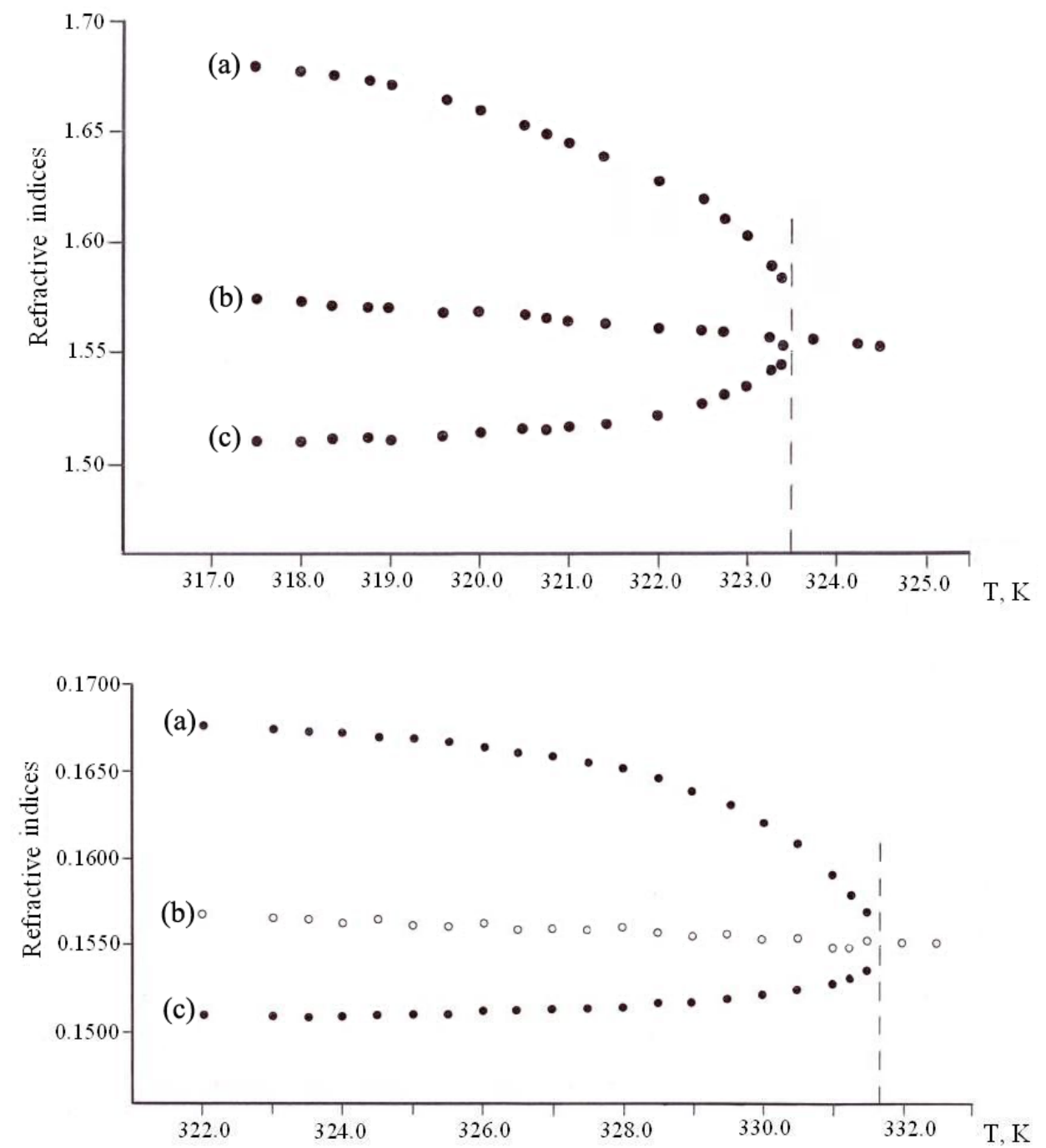

Fig. 4. The temperature dependences of the $n_{\mathrm{e}}(\mathrm{a})$, $n$ (b) and $n_{\mathrm{o}}$ (c) refractive indexes for 10CB.
Fig. 5. The temperature dependences of the $n_{\mathrm{e}}(\mathrm{a})$, $n$ (b) and $n_{\mathrm{o}}$ (c) refractive indexes for $12 \mathrm{CB}$. 
refractive indices is due to decreased orderedness, which results in a decrease of the optical anisotropy in the nematic mesophase as compared with the smectic A mesophase.

The temperature dependences of $\alpha_{\mathrm{e}}$ and $\alpha_{0}$ for the liquid crystals under investigation are presented in Figs. 6-8. As seen in these figures, the temperature dependences of $\alpha_{\mathrm{e}}$ and $\alpha_{0}$ exhibit a similar behaviour for $8 \mathrm{CB}, 10 \mathrm{CB}$ and $12 \mathrm{CB}$. These figures demonstrate that as temperature increases, $\alpha_{\mathrm{e}}$ decreases and $\alpha_{0}$ increases. A break in $\alpha_{\mathrm{e}}$ and $\alpha_{0}$ is observed at the SmA-N and N-I phase transitions for $8 \mathrm{CB}$ and at the SmA-I phase transitions for $10 \mathrm{CB}$ and $12 \mathrm{CB}$. In the isotropic liquid state $\alpha_{\mathrm{o}}=0$ and $\alpha_{\mathrm{e}}=0$ takes place. That fact indicates disappearance of the principal polarizabilities of the liquid crystalline mesophase in the directions of the ordinary and extraordinary rays. Using the $\alpha_{\mathrm{e}}$ and $\alpha_{0}$ values, the average polarizability $\alpha_{\text {ave }}$ was determined by

$$
\alpha_{\text {ave }}^{2}=\frac{\alpha_{\mathrm{e}}^{2}+2 \alpha_{0}^{2}}{3} \text {. }
$$

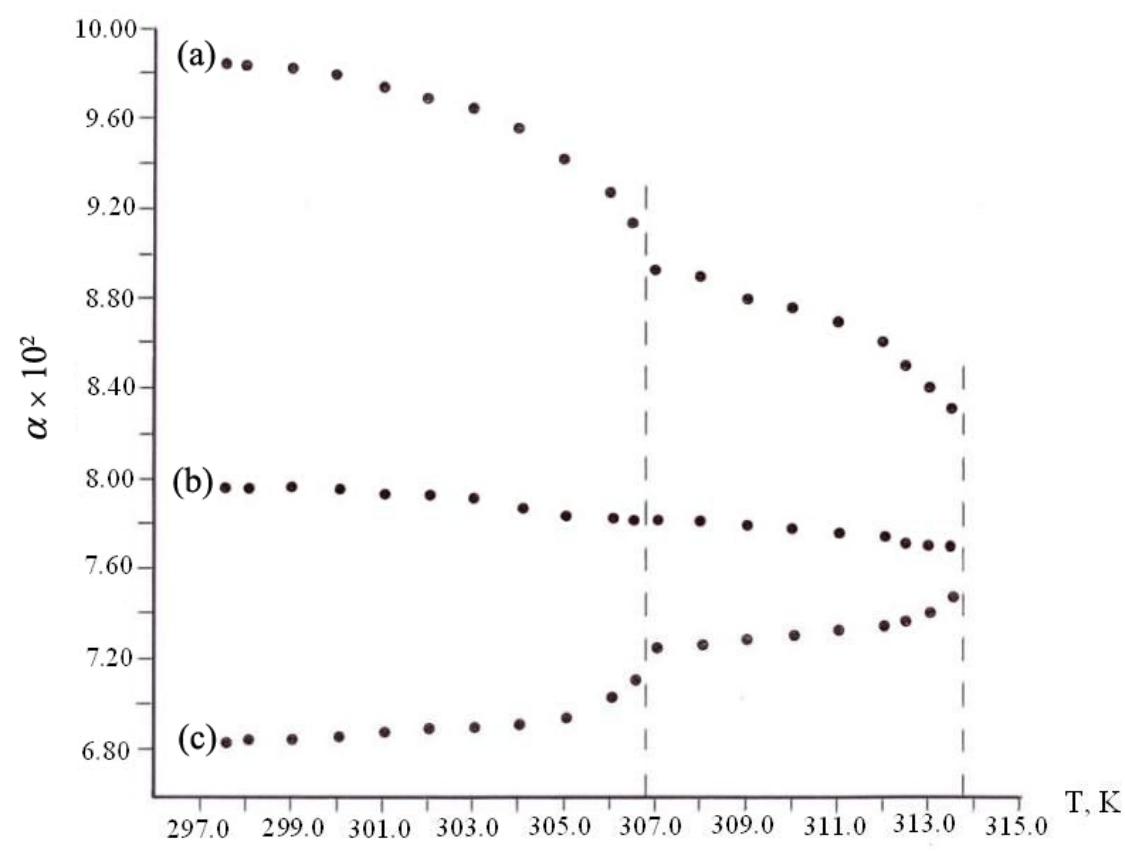

Fig. 6. The temperature dependences of $\alpha_{\mathrm{e}}(\mathrm{a}), \alpha_{\text {ave }}$ (b) and $\alpha_{0}(\mathrm{c})$ for $8 \mathrm{CB}$.

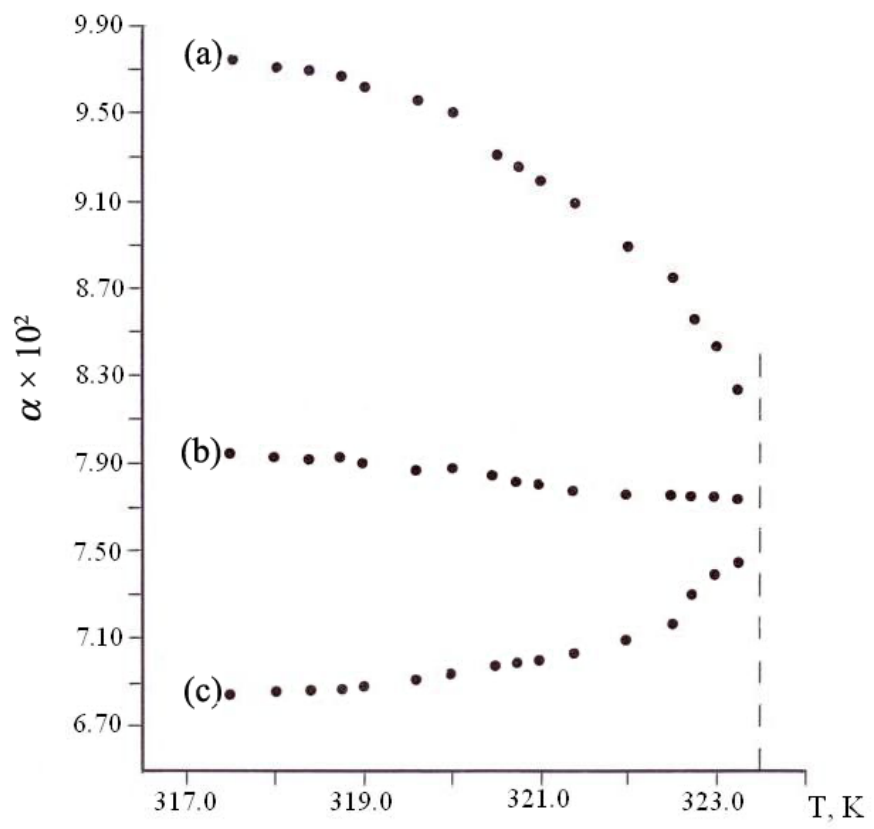

Fig. 7. The temperature dependences of $\alpha_{\mathrm{e}}$ (a), $\alpha_{\text {ave }}$ (b) and $\alpha_{0}$ (c) for 10CB. 


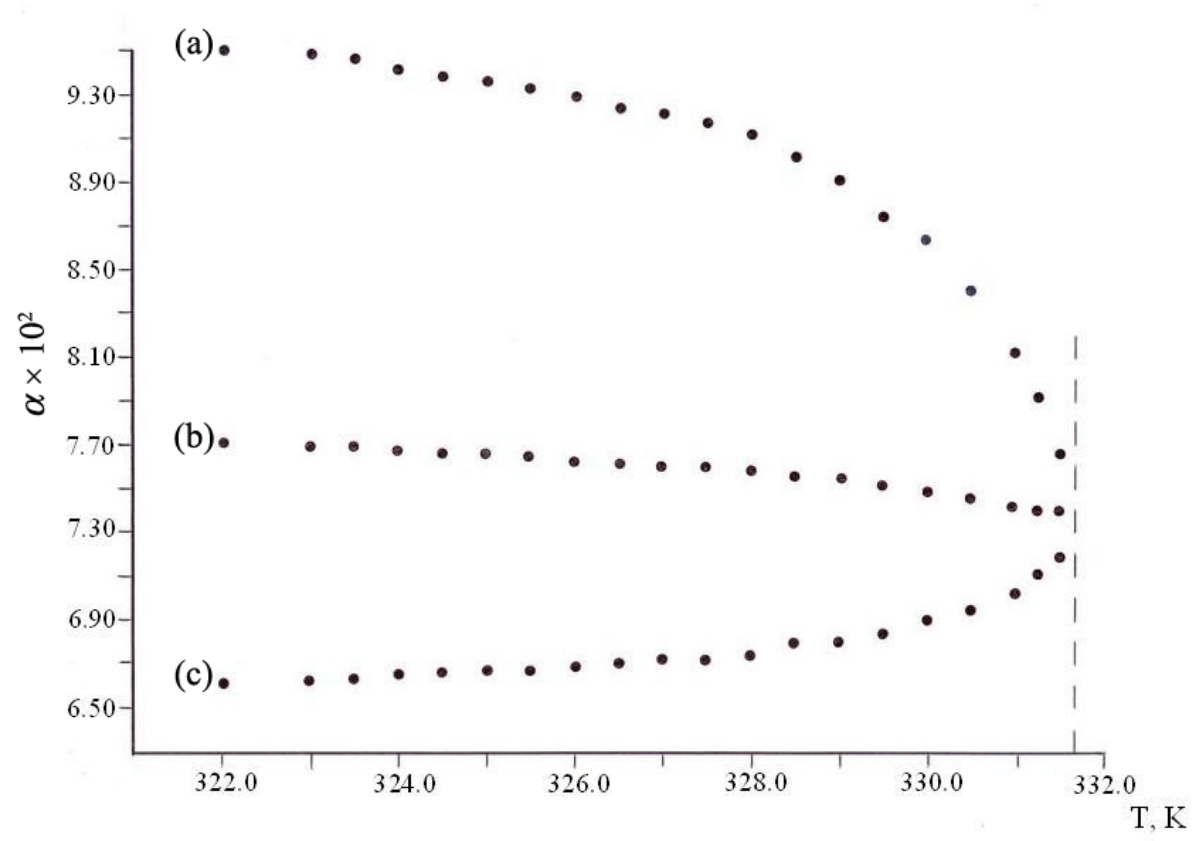

Fig. 8. The temperature dependences of $\alpha_{\mathrm{e}}$ (a), $\alpha_{\text {ave }}$ (b) and $\alpha_{0}$ (c) for 12CB.
As seen in Figs. 6-8, $\alpha_{\text {ave }}$ is almost constant in both nematic and smectic $\mathrm{A}$ mesophases for the liquid crystals under investigations. Such behaviour of $\alpha_{\text {ave }}$ corresponds to the temperature behaviour of the optical isotropic parameter, i. e. the behaviour of $n$.

In Fig. 9, the temperature dependences of $\alpha_{\mathrm{eg}}$ are presented. As seen in this figure, it is found that $\alpha_{\mathrm{eg}}$ fluently increases with a temperature increase in the nematic mesophase region for $8 \mathrm{CB}$ and in the smectic A mesophase region for $8 \mathrm{CB}, 10 \mathrm{CB}$ and $12 \mathrm{CB}$. The value of $\alpha_{\text {eg }}$ aspires to unity in the isotropic liquid state for these liquid crystals. When $\alpha_{\mathrm{eg}}$ reaches unity, this means that in this temperature region there is not any orientational order in liquid crys- talline material and the value of the order parameter is therefore zero. Such behaviour of $\alpha_{\mathrm{eg}}$ is related with the fact that differences between $n_{\mathrm{e}}$ and $n_{\mathrm{o}}$, and accordingly the $\Delta n$ value are decreased with an increase in temperature (Eq. 9). A similar behaviour of $\alpha_{\text {eg }}$ was predicted in [43] and was also observed by various researches for different liquid crystalline materials in [15, 28, 41].

The behaviour of $\Delta n$, as the behaviour of other tensorial parameters (e. g. anisotropy of dielectric properties, anisotropy of viscosity, etc.), corresponds to the behaviour of the order parameter. As it is noted in [33], the measurements of $\Delta n=\Delta n(T)$ provide a more direct method to obtain a fairly ac-

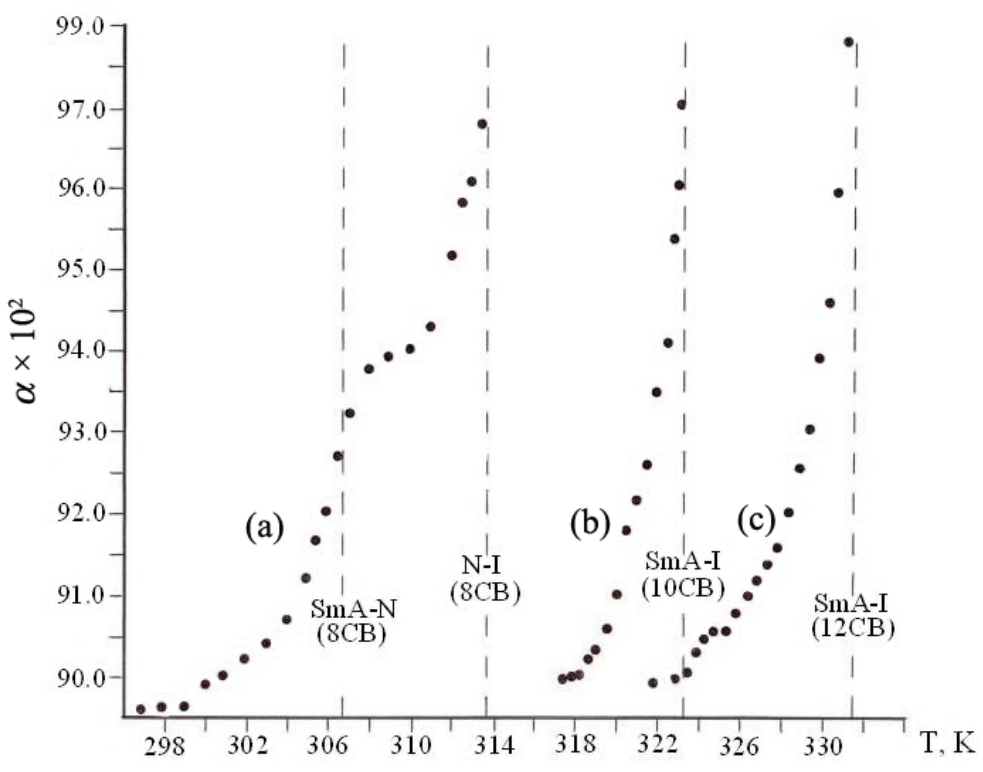

Fig. 9. The temperature dependences of $\alpha_{\text {eg }}$ for 8CB (a), 10CB (b) and 12CB (c). 
curate value of the order parameter. Therefore, a behaviour of the order parameter in a large number of liquid crystalline materials has been reported, using the $\Delta n$ data [ [月, 8, 15, 20, 24, 31, 35, 40, 45-47]. In Fig. 10, the temperature dependences of the birefringence for $8 \mathrm{CB}, 10 \mathrm{CB}$ and $12 \mathrm{CB}$ are shown. From these figures it is observed that longer homologues display distinctly smaller optical anisotropy. This fact may be attributed to the higher clearing temperature for the longer homologues. Such peculiarity for other homologues was also observed in various liquid crystalline materials in [1, 15, 20]. Besides, as seen in Fig. 10, a fluent decrease of $\Delta n$ with an increase of temperature and jump-like change of this parameter in the region of the $\mathrm{SmA}-\mathrm{N}$ phase transition takes place for $8 \mathrm{CB}$.

As it is known, the character of the $\mathrm{SmA-N}$ phase transition is determined by the orientational order parameter $Q$ and translational order parameter $|\psi|$. The parameter $|\psi|$ describes correlation of the gravity centers of molecules in smectic layers [30, 48, 49]. In the nematic mesophase $|\psi|=0$ and in the smectic mesophase $|\psi| \neq 0$ takes place. Deviation of the order parameter $Q(T)$ in the smectic A mesophase from this parameter $Q_{0}(T)$ in the nematic mesophase is determined by

$$
\delta Q=Q-Q_{0}=\kappa C|\psi|^{2} .
$$

Here $\kappa$ is a function, which is dependent on difference between temperatures of the SmA-N $\left(T_{A N}\right)$ and N-I $\left(T_{N I}\right)$ phase transitions; $C$ is a constant. For the first order SmA-N phase transition $\delta Q \neq 0$, for the second order $\mathrm{SmA}-\mathrm{N}$ phase transition $\delta Q=0$. In the microscopic theory, the numerical criterion for the SmA-N phase transition is presented [30, 48, 50].
This criterion is connected with the above-mentioned $T_{A N}$ and $T_{N I}$ transition temperatures. For $\frac{T_{A N}}{T_{N I}}=\beta>0.87$ the first order transition, for $\frac{T_{A N}}{T_{N I}}=\beta<0.87$ the second order transitions take place. As it is seen in the Table, the ratio between $T_{A N}$ and $T_{N I}$ is as $\frac{T_{A N}}{T_{N I}} \approx 0.95$ for $8 \mathrm{CB}$. Thus, jump-like behaviour of the $\Delta n$ and value of the

$\beta$ criterion indicates the first order transition between smectic A and nematic mesophases in 8CB.

As seen in Fig. 10, the abrupt changes of the $\Delta n$ in the region of the SmA-I phase transition take place for $10 \mathrm{CB}$ and $12 \mathrm{CB}$. Such behaviour of $\Delta n$ vs. temperature indicates the first order SmA-I phase transition. A similar behaviour of $\Delta n$ for the first order transition has been also observed in 447,48 . The direct SmA-I phase transition has attracted increasing attention because that is transition from the layering organized structure to the disordered physically isotropic state. Peculiarities of the SmA-I phase transition have been experimentally studied by various scientists for different liquid crystalline materials in [22, 51-58]. In these works the SmA-I phase transition as the first order transition was found. Besides, this transition is more strongly first order than the N-I transition, which is known to be a sufficiently weak first order transition. The SmA-I phase transition within a phenomenological Ginzburg-Landau and Landau-de Gennes approaches has been theoretically investigated in [59-66]. In this work the above-mentioned transition is found to be the strong first order. Besides, in [66] this result is compared with a wide variety of phenomena including effects of nonmesogenic impurities and electric field,

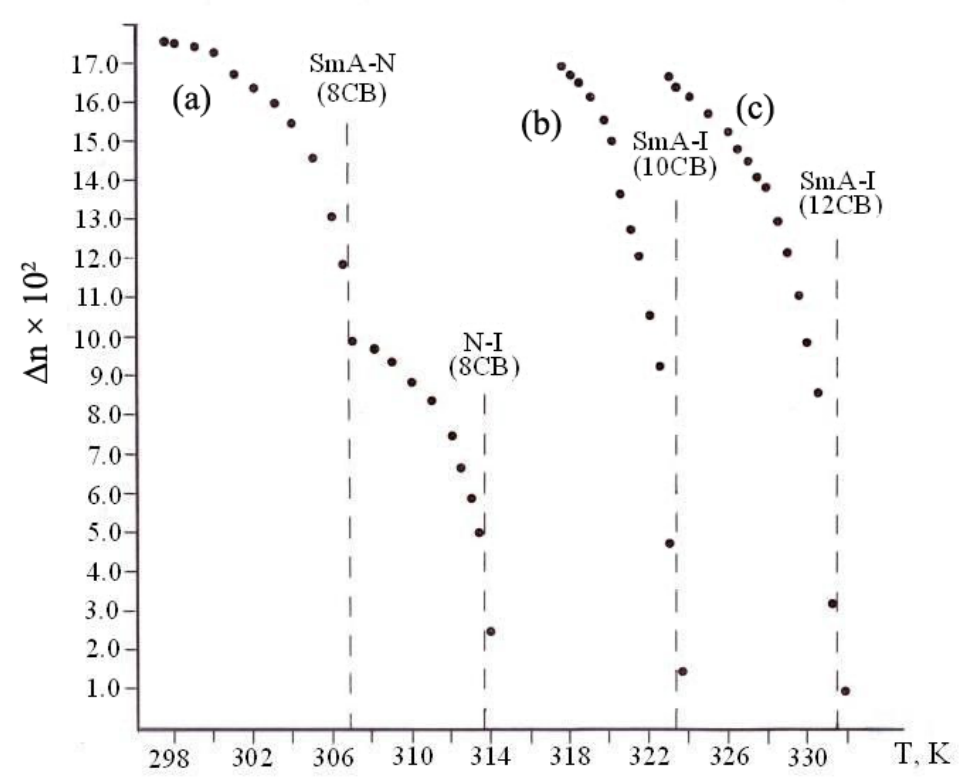

Fig. 10. The temperature dependences of $\Delta n$ for $8 \mathrm{CB}$ (a), 10CB (b) and 12CB (c). 
hydrodynamics, fluctuations, and the elastic theory of liquid crystalline elastomers. Thus, the results, obtained in the present study, are in well conformity with experimental results, obtained in [22, 51-58], and with theoretical predictions, obtained in [59-66].

\section{Conclusions}

In this work, the thermotropic, refracting and thermooptical properties of the 4-n-alkyl-4'-cyanobiphenyls $(\mathrm{n}=8,10,12)$ have been studied. Investigations have been carried out for the planar alignment, homeotropic alignment and non-alignment samples. Results showed that the longer homologues of the 4-n-alkyl-4'cyanobiphenyls displayed distinctly smaller optical anisotropy. Namely, the maximum birefringence values are as $\Delta n=0.1760$ for $8 \mathrm{CB}, \Delta n=0.1700$ for $10 \mathrm{CB}$ and $\Delta n=0.1680$ for $12 \mathrm{CB}$. This fact may be attributed to the higher clearing temperature for the longer homologues $(T=306.8 \mathrm{~K}$ for $8 \mathrm{CB}, T=317.1 \mathrm{~K}$ for $10 \mathrm{CB}$ and $T=321.3 \mathrm{~K}$ for $12 \mathrm{CB})$.

Investigations show that the extraordinary refractive index $\left(n_{\mathrm{e}}\right)$ and the $\alpha_{\mathrm{e}}$ principal polarizability decrease sharply while the ordinary refractive index $\left(n_{\mathrm{o}}\right)$ and the $\alpha_{\mathrm{o}}$ principal polarizability increase slightly as the temperature increases. The breaks in $n_{\mathrm{e}^{\prime}} \alpha_{\mathrm{e}}$ and $n_{\mathrm{o}^{\prime}}$ $\alpha_{0}$ are observed at the SmA-N and N-I phase transitions for $8 \mathrm{CB}$ and at the SmA-I phase transitions for $10 \mathrm{CB}$ and $12 \mathrm{CB}$. These breaks are related with abrupt changes in the optical anisotropy of liquid crystalline mesophase and in the polarizability of molecules in the phase transition regions.

The temperature behaviour of $n$ and $\alpha_{\text {ave }}$ indicates thermal stability of the refractivity and polarizability of $8 \mathrm{CB}, 10 \mathrm{CB}$ and $12 \mathrm{CB}$. In this work the extrapolated average refractive index $\left(n_{\text {ave }}\right)$ has been obtained by the extrapolation of the refractive index of isotropic liquid $\left(n_{\text {iso }}\right)$ into the nematic and smectic A mesophases for $8 \mathrm{CB}$ and into the smectic $\mathrm{A}$ mesophase for $10 \mathrm{CB}$ and $12 \mathrm{CB}$. The results, which have been obtained by extrapolating, entirely coincide with the results, which have been obtained by the refractometric method.

The character of temperature changes of $\Delta n$ in the phase transition coincides with the character of temperature changes of the order parameter. The $\Delta n=\Delta n(T)$ dependences indicate the first order SmA$\mathrm{N}$ and $\mathrm{N}-\mathrm{I}$ phase transitions in $8 \mathrm{CB}$ and the first order SmA-I phase transitions in 10CB and 12CB.

\section{Acknowledgements}

Part of this work has been carried out in the Department of Medical and Biological Physics of Azerbaijan Medical University, Baku.

\section{References}

[1] L.M. Blinov and V.G. Chigrinov, Electrooptic Effects in Liquid Crystal Materials (Springer Verlag, New York, 1993).

[2] M.A. Anisimov, Critical Phenomena in Liquids and Liquid Crystals (Gordon and Breach Science Publishers, New York-London, 1991).

[3] P. Yeh and C. Gu, Optics of Liquid Crystals Displays (Wiley Publishing, London-New York, 2009).

[4] I.C. Khoo, Liquid Crystals (Wiley Publishing, London-New York, 2007).

[5] H.S. Subramhanyam, C.S. Prabha, and D. Krishnamurti, Optical anisotropy of nematic compounds, Mol. Cryst. Liq. Cryst. 28, 201-215 (1974).

[6] A. Nesrullajev, A.S. Sonın, and A.Z. Rabinovich, Study of the character of smectic A-nematic phase transitions in cyanobiphenyl mixtures, Crystallography 25, 435-439 (1980) [in Russian].

[7] M. Mitra, S. Gupta, R. Paul, and S. Paul, Determination of orientational order parameter from optical studies for a homologous series of mesomorphic compounds, Mol. Cryst. Liq. Cryst. 199, 257-266 (1991).

[8] M.S. Zakerhamidi, Z. Ebrahimi, H. Tajalli, A. Ghanadzadeh, M. Modhadam, and A. Ranjkesh, Refractive indices and order parameters of some tolane-based nematic liquid crystals, J. Mol. Liq. 157, 119-124 (2010).

[9] K. Bhowmick, A. Mukhopadhyay, and C.D. Mukherjee, Texture and optical studies of the mesophases of cyanocyclohexyl cyclohexanes, Phase Transit. 76, 671-682 (2003).

[10]M.F. Vuks, Determination of optical anisotropy of aromatic molecules from double refraction in crystals, Opt. Spectrosc. 20, 361-368 (1966) [in Russian].

[11]M.F. Vuks, Electrical and Optical Properties of Molecules and Condensed Matter (Leningrad University Publishing House, Leningrad, 1984) [in Russian].

[12] H.E.J. Neugebauer, Clausius-Mossotti equation for certain types of anisotropic crystals, Can. J. Phys. 32, 1-8 (1954).

[13]H.S. Subramhanyam and D. Krishnamurti, Polarization field and molecular order in nematic liquid crystals, Mol. Cryst. Liq. Cryst. 22, 239-248 (1973).

[14]D. Bhuyan, P. Pardhasarathi, K.N. Singh, D. Mathavi Latha, P.V. Datta Prasad, P.R. Alapati, and V.G.K.M. Pisipati, Study of molecular polarizabilities and orientational order parameter in the nematic phase of 6.0120.6 and 7.0120.7, World J. Condens. Matter. Phys. 1, 167-174 (2011).

[15] S.S. Sastry, T.V. Kumari, K. Mallika, B.G. Sankara Rao, S.-T. Ha, and S. Lakshminarayana, Order parameter studies on EPAP alcanoate mesogens, Liq. Cryst. 39, 295-301 (2012). 
[16] I. Haller, Thermodynamic and static properties of liquid crystals, Progr. Solid State Chem. 10, 103118 (1975).

[17]I. Haller, H.A. Huggins, H.R. Lilienthal, and T.R. McGuire, Order-related properties of some nematic liquids, J. Phys. Chem. 77, 950-954 (1973).

[18]I. Haller, H.A. Huggins, and M.H. Freiser, On the measurement of indices of refraction of nematic liquid crystals, Mol. Cryst. Liq. Cryst. 16, 53-59 (1972).

[19]M. Sharma, C. Kaur, J. Kumar, K.C. Singh, and P.C. Jain, Phase transformations in some homologues of 4-n-alkyl-4'-cyanobiphenyls investigated by positron annihilation spectroscopy, J. Phys. Condens. Matter. 13, 7249-7258 (2001).

[20]I. Chirtoc, M. Chirtoc, C. Glorieux, and J. Thoen, Determination of the order parameter and its critical exponent for $\mathrm{nCB}(\mathrm{n}=5-8)$ liquid crystals from refractive index data, Liq. Cryst. 31, 229-240 (2004).

[21]R.-P. Pan, T.-R. Tsai, C.-Y. Chen, C.-H. Wang, and C.-L. Pan, The refractive indices of nematic liquid crystal 4'-n-pentyl-4-cyanobiphenyl in the THz frequency range, Mol. Cryst. Liq. Cryst. 409, 137-144 (2004).

[22]G. Chahine, A.N. Kityk, N. Demarest, F. Jean, K. Knorr, P. Huber, R. Lefort, J.-M. Zanotti, and D. Morineau, Collective molecular reorientation of a calamitic liquid crystal (12CB) confined in alumina nanochannels, Phys. Rev. E 82, 011706 (2010).

[23]T. Nose, S. Sato, K. Mizuno, J. Bae, and T. Nozukido, Refractive index of nematic liquid crystals in the submillimeter wave region, Appl. Opt. 36, 63836387 (1997).

[24]S.K. Sarkar, P.C. Barman, and M.K. Das, Determination of optical birefringent and orientational order parameter of four members of alkyl cyanobiphenyls using high resolution temperature scanning technique, Int. J. Res. Appl. Nat. Soc. Sci. 1, 1-8 (2013).

[25]S. Chandrasekhar and N.V. Madhusudana, Orientational order in p-azoxyanisole, p-azoxyphenetole and their mixtures in nematic phase, J. Phys. Colloques 30, C4-24 (1969).

[26]N.V. Madhusudana and R. Pratibha, Elasticity and orientational order in some cyanobiphenyls: Part IV. Reanalysis of the data, Mol. Cryst. Liq. Cryst. 89, 249-257 (1982).

[27]T.N. Soorya, S. Gupta, A. Kumar, S. Jain, V.P. Arora, and B. Bahadur, Temperature dependent optical property studies of nematic mixtures, Indian J. Pure Appl. Phys. 44, 524-531 (2006).

[28]A. Kumar, Determination of orientational order and effective geometry parameter from refractive indices of some nematics, Liq. Cryst. 40, 503-510 (2013).

[29]P.V. Adomenas, A.N. Nesrullajev, B.I. Ostrovski, A.Z. Rabinovich, and A.S. Sonin, The change of the character of smectic A-nematic phase transition in binary mixtures of liquid crystals, Phys. Solid State 21, 2492-2496 (1979) [in Russian].

[30]A.S. Sonin, Introduction to the Physics of Liquid Crystals Science Publishing House, Moscow, 1983) [in Russian].

[31]J.C. Tolédano and P. Tolédano, The Landau Theory of Phase Transitions (World Scientific, Singapore, 1987).

[32] M.A. Anisimov, Critical phenomena in liquid crystals, Mol. Cryst. Liq. Cryst. A162, 1-96 (1988).

[33]R. Manohar and J.P. Shukla, Refractive indices, order parameter and principal polarizability of cholesteric liquid crystals and their homogeneous mixtures, J. Phys. Chem. Solids 65, 1643-1650 (2004).

[34]W.H. de Jeu, Physical Properties of Liquid Crystalline Materials (Gordon and Breach, New York-London-Paris, 1980).

[35] A. Prasad and M.K. Das, Optical birefringence studies of a binary mixture with the nematicsmectic $A_{d}-$ re-entrant nematic phase sequence, J. Phys. Condens. Matter. 22, 1-7 (2010).

[36] M. Ramakrishna, N. Rao, P.V. Datta Prasad, and V.G.K.M. Prisipati, Orientational order parameter in alkoxy benzoic acids - Optical studies, Mol. Cryst. Liq. Cryst. 528, 49-63 (2010).

[37]J.L. Kumari, P.V.D. Prasad, D.M. Latha, and V.G.K.M. Pisipati, Orientational order parameter estimated from molecular polarizabilities - an optical study, Phase Trans. 85, 52-64 (2012).

[38] P.V. Datta Prasad and V.G.K.M. Pisipati, Simple, accurate and low cost optical techniques for the measurement of 1. Birefringence in liquid crystal and 2. Variation of the angle of the small angled prism with temperature, Mol. Cryst. Liq. Cryst. 511, 102-111 (2009).

[39] W. Kuczynski, B.J. Zywicki, and J. Malecki, Determination of orientational order parameter in various liquid crystalline phases, Mol. Cryst. Liq. Cryst. 381, 1-19 (2002).

[40]A.K. Srivastava, R. Manohar, and J.P. Shukla, Refractive indices, order parameter and principal polarizability of cholesteric liquid crystals and their mixtures, Mol. Cryst. Liq. Cryst. 454, 225-234 (2006).

[41]S.S. Sastry, T.V. Kumari, S.S. Begum, and V.V. Rao, Investigations into effective order geometry in a series of liquid crystals, Liq. Cryst. 38, 277-285 (2011).

[42]M.D. Gupta, A. Mukhopadhyay, and K. Czuprynski, Optical properties of two liquid crystal compounds: a comparative study, Phase Trans. 83, 284-292 (2010).

[43] C. Satiro and F. Moraes, On the deflection of light by topological defects in nematics liquid crystals, Eur. Phys. J. 25, 425-429 (2008).

[44]C. Satiro and F. Moraes, Lensing effects in a nematic liquid crystal with topological defects, Eur. Phys. J. E 20, 173-178 (2006). 
[45] M.M.M. Abdoh, S.N.C. Shivaprakash, and J.S. Prasad, Orientational order in the nematogenic homologous series trans-4-alkyl (4-cyanobiphenyl)cyclohexane, J. Chem. Phys. 77, 2570-2576 (1982).

[46]A. Kumar, Determination of orientational order and effective geometry parameter from refractive indices of some nematics, Liq. Cryst. 40, 503-510 (2013).

[47]W.H. de Jeu and P. Bordewijk, Physical studies of nematic azoxybenzenes. II. Refractive indices and the internal field, J. Chem. Phys. 68, 109-115 (1978).

[48] W.L. McMillan, Simple molecular model for the smectic A phase of liquid crystals, Phys. Rev. A 4, 1238-1246 (1971).

[49]P.G. de Gennes and J. Prost, The Physics of Liquid Crystals (Oxford Science Publications, OxfordLondon, 2003).

[50]P.G. de Gennes, The Physics of Liquid Crystals (Clarendon Press, Oxford-London, 1973).

[51]G. Chahine, A.N. Kityk, K. Knorr, R. Lefort, M. Guendouz, D. Morineau, and P. Huber, Criticality of an isotropic-to-smectic transition induced by anisotropic quenched disorder, Phys. Rev. E 81, 031703 (2010).

[52]B.M. Ocko, A. Braslau, P.S. Pershan, J. Als-Nielsen, and M. Deursch, Quantized layer growth at liquidcrystal surfaces, Phys. Rev. Lett. 57, 94-97 (1986).

[53]A. Nesrullajev and Ş. Oktik, Induced changes of smectic A-isotropic liquid phase transition peculiarities: Effect of surfaces, Mod. Phys. Lett. B 14, 821-833 (2006).

[54]N. Yilmaz-Canli, A. Nesrullajev, and B. Bilgin Eran, Synthesis, mesomorphic and physical properties of two new analogs of Schiff's base with an alkenic terminal chains, J. Mol. Str. 990, 79-85 (2011).

[55]A. Drozd-Rzoska, Influence of measurement frequency on the pretransitional behaviour of the nolinear dielectric effect in the isotropic phase of liquid crystalline materials, Liq. Cryst. 24, 835-840 (1998).

[56] A. Drozd-Rzoska, S.J. Rzoska, and J. Ziolo, Quasicritical behaviour of dielectric permittivity in the isotropic phase of smectogenic n-cyanobiphenyls, Phys. Rev. E 61, 5349-5344 (2000).

[57] S.G. Polushin, V.B. Rogozhin, E.I. Ryumtsev, and A.L. Lezov, The kerr effect in the vicinity of the transition from the isotropic to smectic A phase, Russ. J. Phys. Chem. 80, 1016-1020 (2006).

[58] S.J. Rzoska, P.K. Mukherjee, and M. Rutkowska, Does the characteristic value of the discontinuity of the isotropic-mesophase transition in $n$-cyanobiphenyls exist? J. Phys. Condens. Matter. 24, 375101(1-10) (2012).

[59] K.P. Sidgel and G.S. Innachione, Study of the isotropic to smectic-A phase transition in liquid crystal and acetone binary mixtures, J. Chem. Phys. 133, 174501 (2010).

[60]M. Olbrich, H.R. Brand, H. Finkelmann, and K. Kavasaki, Fluctuations above the smectic-Aisotropic transition in liquid crystalline elastomers under external stress, Europhys. Lett. 31, 281-286 (1995).

[61] G. Gordoyiannis, L.F.V. Pinto, M.H. Godinho, C. Glorieux, and J. Thoen, High-resolution calorimetric study of the phase transitions of tridecylcyanobiphenyl and terradecylcyanobiphenyl liquid crystals, Phase Trans. 82, 280-289 (2009).

[62] H. Pleiner, P.K. Mukherjee, and H.R. Brand, Direct transitions from isotropic to smectic phases, in: Proceedings of the 29th Freiburger Arbeitstagung Flüssigkristalle (2000) pp. 59-62.

[63]H.R. Brand, P.K. Mukherjee, and H. Pleiner, Macroscopic dynamics near the isotropic-smectic-A phase transition, Phys. Rev. E 63, 061708-1 (2001).

[64] P.K. Mukherjee, H. Pleiner, and H.R. Brand, Simple Landau model of the smectic-A-isotropic phase transition, Eur. Phys. J. 4, 293-297 (2001).

[65] P.K. Mukherjee and S.J. Rzoska, Pressure effect on the smectic-A-isotropic phase transition, Phys. Rev. E 65, 051705 (2002).

[66] P.K. Mukherjee, Isotropic to smectic-A phase transition: A review, J. Mol. Str. 190, 99-111 (2013).

\title{
TRIJŲ 4-N-ALKIL-4'-CIANOBIFENILŲ TERMOTROPINĖS, ATSPINDŽIO IR TERMOOPTINĖS SAVYBE்S
}

\author{
A. Nesrullajev \\ Mugla Sitki Koçman universiteto Mokslo fakulteto Fizikos katedra, \\ Kötekli Muğla, Turkija
}

